\title{
Understanding and Supporting Users to Improve Atmosphere of Communication by Kansei Agents
}

\author{
Shigaku Tei*, Tatsuki KAWAGUCHI*, Teck Ceng SIM** and Hisao SHIIZUKA*** \\ * The University of Aizu, 90 Tsuruga Ikkimachi, Aizuwakamatsu, Fukushima, 965-8580, Japan \\ z-cheng@u-aizu.ac.jp / kawaguch@u-aizu.ac.jp \\ ** The University of Aizu, Junior College Division, 1-1 Monden, Ikkimachi, Aizuwakamatsu, Fukushima, 965-8580, Japan \\ tcsim@jc.u-aizu.ac.jp \\ ***SKEL, Shiizuka Kansei Engineering Laboratory, Tokyo, Japan \\ shiizuka@flsi.or.jp
}

\begin{abstract}
In order to maintain smooth human interactions and communications, atmosphere of communication plays an important role besides exchanging information or negotiation among the persons involved in the communication, this paper proposes how Kansei/ Affective agents can detect the atmosphere and support the better communication. We also design a CNN based feasibility experiment as the first step to detect the dialogue state of communication, which is one factor of communication atmosphere.

Keywords: Kansei Communication, Atmosphere of Communication, Situation Theory, Kansei/Affective Agents, Convolution Neural Network (CNN)
\end{abstract}

\section{INTRODUCTION}

In human-to-human communication, in addition to convey information / knowledge logically, there are also Kansei/Affective parts that are ambiguous and understanding with atmosphere of communication. Some people have various experiences and communicate based on the indirect atmosphere depending on the situation. However, someone has been said that "they cannot read the air", which means they cannot catch the meaning through indirect ambiguous expressions

The purpose of this paper is to propose a Kansei/ Affective agent, which can comprehend the atmosphere of communication, and improve communication between human being. The Kansei agents are initial trial to realize concept of affective kansei [1].

In order to realize the purpose, we will solve how to represent and detect the atmosphere, how to understand or recognize the atmosphere, and how to support communication based on the atmosphere recognized.

To this end, the dialogue state, which is one factor to influence the atmosphere, in a specific situation is represented by a two-dimensional image based on situation theory model [2], and we train a Convolutional Neural Network (CNN) using the data of the images, to understand and support communication for the specific situation

As a test case, we develop an ideal situation "family communication at the dining table", describe the dialogue problems between family members, and use the prediction of the actions in that situation as a training target. Feasibility experiment is designed to show it is feasible to recognize the different atmospheres represented by a comic like drawing representation.

\section{MODEL AND DIFINITIONS}

\subsection{The Model of Kansei Communication}

Kansei/Affective atmospheres plays important role in communication [1]. Fig. 1 shows a model of the communication situation, where three people communicate with each other, but it can be generalized more than three people. We assume that each person can speak logically and understand the other person's story, as indicated by bi-direction white arrows. On the other hand, the shape of the cloud around a person shows the affective aspect of the person, such as his/her gestures or non-verbal expression. Although ambiguous verbal can also express the affection, this paper assumes that there is no ambiguous expression of the language in order to simplify the model. Besides communication between persons expressed by logical points represented by the white circle on the situation, a dotted gray circle represents the atmosphere of communication. The atmosphere of the situation is formed by the affective aspect of each of the three, and affects the next action and the next situation. A Kansei/affective agent is used to detect the situation, especially the affective atmosphere of the situation, and make prediction on possible actions which may happen on such a situation, as well as changes 
of the situation. The agent can also provide some hints to improve the atmosphere by directly show something to all the three persons or to some of them as shown with the black arrows.

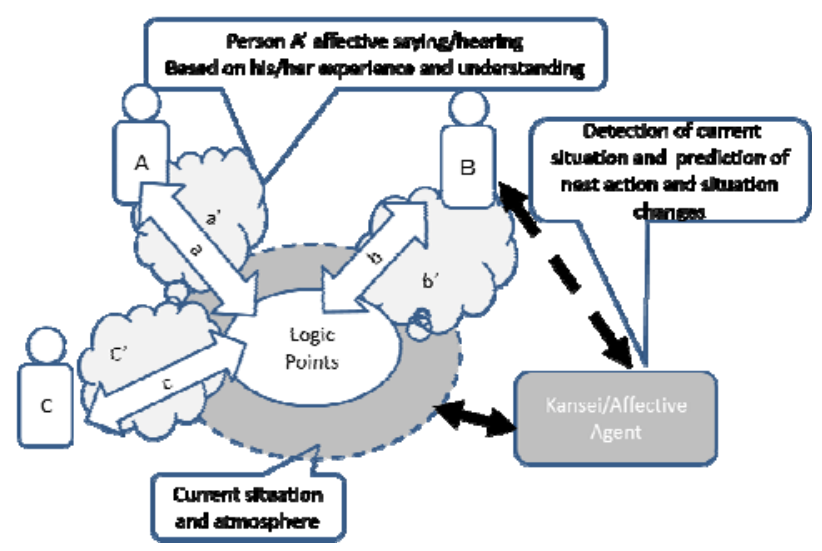

Figure 1: Model of a Communication Situation

\subsection{Situation Theory based Atmosphere Description}

Situation theory [2] is a framework that describes various situations in four elements. That is, individuals, their attributes, relationships between individuals, and a spatial-temporal location. Furthermore, actions and changes of attributes and relationships can also be defined on each situation. Based on the situation theory, we presume that the atmosphere of communication under influence of the emotional mood of each individual, relationships between individuals, dialogue state, and the surrounding environment. In this paper, we define human communication issues by adding affective atmosphere on the dialogue state.

\subsection{Problems in Communication of Family When Eating at the Dining Table}

Even in families, there are various communication problems due to differences in values such as differences in thinking and feelings between parents and children, between married couples, and between brothers. For example, while parents repeatedly say "study", "study", "poor grades", the child would not take any action and no matter what the parent says, the child does not respond. Also, the relationship between brothers deteriorates due to various reasons, and always quarrels when eating together at the dining table, and the couples have been disagreeing with each other for many years and have met each other in a mode where they do not talk each other deeply.

In case of father and son's interaction in the family, depending on the age or any other social factors, it sometimes difficult to communicate, and positive or negative atmosphere will be created. For instance, father and son are having a conversation about the son's final grade at a family dining table. In case of Fig. 2(a) as a positive atmosphere, both father and son are trying to maintain the conversation with good and smooth dialogue. They talk each other interactively, and participate the communication with facing each other. For instance, father was wondering about the bad grade on the mathematics, and asking son about the reasons. Although son had studied really hard, son had a headache during final exam. Dad understood the situation, and encourage son's work hard that shows smooth communication with positive atmosphere.

On the other hand, Fig.2 (b) is a negative atmosphere, father is approaching his son by talking one-way, and son cannot take any actions neither communication nor dialogues. With the same situation above, father complained why he was not able to take care of his health condition before the exam, asked son about other exams. Son was not comfortable discussing the matter, and deny the conversation with his dad that makes not smooth communication with a negative atmosphere.

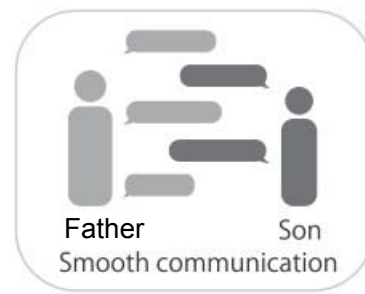

(a) Positive Atmosphere

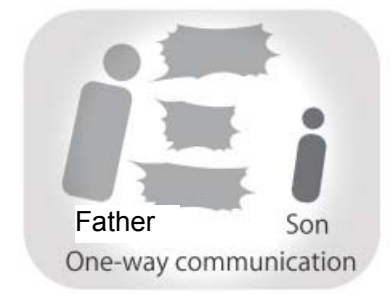

(b) Negative Atmosphere Figure 2: Atmosphere of Communication

\section{DESIGN OF THE SYSTEM (KANSEI AGENT) 3.1 Overview of the System}

As shown in Fig. 3, the total system (Kansei agent) consists of (a) a detection and recognition module and (b) a support module. The module (a) further has four sub-modules to detection and recognition of

1) Everyone's emotion, such as delight, anger, sorrow and pleasure emotions, and etc.

2) Relation between individuals, such as parent-child, brothers, teacher-students, etc.

3) Dialogue state, and

4) Environment, such as noisy, temperature, and smell, which may influent the atmosphere

There are some technologies to analyze face expressions, the tone of voices etc. Relation information can be grasped from a profile of each user or collected from SNS. Physical environmental information can be acquired by various sensors. In this paper, we mainly discuss the dialogue state detection and recognition. 


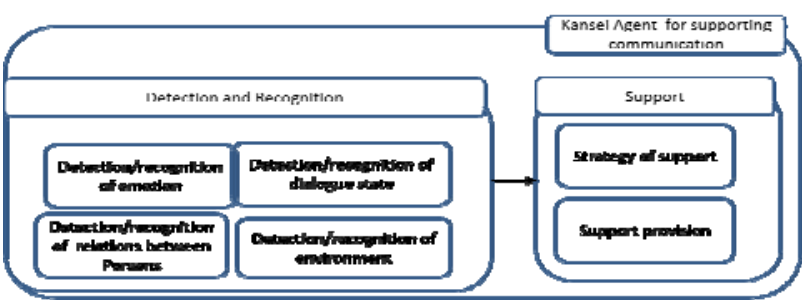

Figure 3: Structure of the System

As shown in Fig. 4, the sub-module of the dialog state based support consists of an input module, a processing module, and an output module. The input module detects the state of human users, such as the number of utterances, the tone, and the like using a camera, a microphone, or the like. And it creates data that reflects the atmosphere based on the detected raw data with pre-processing. The processing module trains a deep learning network $(\mathrm{CNN}$ in this paper) using the input data and detects communication smoothness. For further support and prediction, only CNN isn't enough, RNN and BERT, and GAN as well as new type of deep learning are necessary.

It is also desired if the situation is identified, the next possible action is predicted, or a hint is given to the users who are communicating, so as to improve the communication atmosphere. Kansei agents might keep the profiles and communication logs in the past to know their users' attributes and preferences to help the communication.

The output module uses a variety of possible devices, such as a smartphone, a screen on a table, a vibration, etc., to inform the users communicating with each other about the atmosphere and predicted actions, in order to make improvements and be ready to responses to the actions

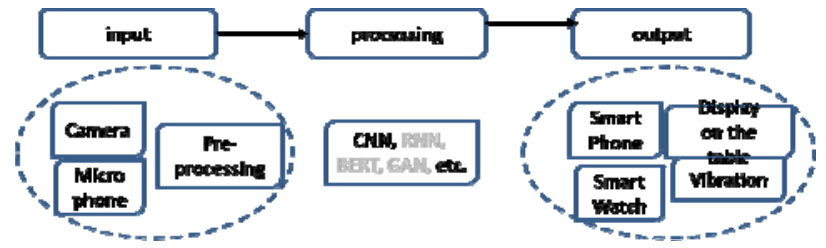

Figure 4: Overview of the Dialog State based Support

\subsection{Data Preparation and Training}

Human-to-human communication captures the Kansai part in various forms, such as linguistic and non-verbal expressions, the flow of speech, facial expressions, and voice tones. As another approach, comic books and picture books express human feelings and the atmosphere of communication with very few words. In this paper, as a first step in realizing the support of Kansai Communication by Kansai Agents, we use pictures/images for representing Kansei dialogues state of atmosphere. Fig. 2 shows an example. Fig. 2 (a) shows that the communication is performed well. In Fig. 2 (b), one person is talking unilaterally, and the other person is silent and does not speak a word.

When talking a lot, the number of utterances and the length of time are measured from the voice detected by a microphone, and expressed by the size and number of speech balloons. As first try, hundreds of such images are prepared and used as data for training.

\subsection{Structure of the network}

There are various methods for processing human emotions, such as image recognition, voice recognition, and natural language processing. In this paper, as a first step, we use CNN to recognize the image data described above.

\section{SUMMARY AND FUTURE WORKS}

In this paper, we describe how the atmosphere of communication between humans is expressed, and we are trying a feasibility experiment of atmosphere detection using CNN based on the expression. In the future, we plan to increase the amount of data further, expand the variety of atmosphere expressions based on situation theory, and further conduct experimental simulations. On the other hand, even in the same atmosphere, each person feels differently. CNN alone is not enough to detect and support each person's sensitivity. We would like to try to simulate various human sensibility parts by combining inductive, deductive, free, abduction, etc. using Innovation Tetra as proposed in [1][3][4].

\section{ACKNOWLEDGMENTS}

The authors would like to thanks Prof. Xing Li and Prof. Jian Chen with the University of Aizu for their advices, and Mr. Ryo Endo, a graduate student of the University of Aizu for his support on the feasibility study.

\section{REFFERENCES}

[1] Hisao Shiizuka and Shigaku Tei, Concept of Artificial Kansei - Challenge to New Artificial Intelligence-, ISASE2020

[2] Jon Barwise and John Perry: Situations and Attitudes, CSLI Publications, pp.46-68,1999

[3] Hisao Shiizuka: Innovation Tetra - Toward the innovation engineering in Era of Kansei 3.0, Proceedings of 2015 Annual Conference of Japan Society of Kansei Engineering, H42, 2015

[4] Hisao Shiizuka: Framework of Innovation Engineering- "Play-Sensibility- Design" Drives Inference Process-, Proceedings of 2016 Spring Conference of Japan Society of Kansei Engineering, WD1-2, 2016 